\title{
LUMINOTÉCNICA - EFICIÊNCIA ENERGÉTICA
}

\section{ILLUMINATION}

\author{
Guilherme Camelo, Breno Monteiro, Marcelo Faleiro, \\ Rodrigo Lincoln, Jurandir Agostinho, Adnan Oliveira, Leonardo Leite
}

\author{
Centro Universitário de Belo Horizonte - UniBH, Belo Horizonte, MG \\ guilherme.camelo@yahoo.com.br; breno.souza.monteiro@vale.com; \\ mfaleiro@gmail.com; rodrigomlincoln@yahoo.com.br; \\ fit.agostinho@gmail.com; adnanoliveira@ig.com.br; \\ leonardo.leite@prof.unibh.br
}

\begin{abstract}
Resumo - A importância das energias renováveis e eficiência energética no cenário mundial é cada vez maior, principalmente no que diz respeito ao consumo e meio ambiente. Alguns aspectos importantes como temperatura de cor das lampâdas, rendimento das luminárias, rendimento do reator utilizado e eficiência luminosa das lâmpadas são de extrema importância para um projeto que visa obter resultados voltados para a eficiência energética. Este artigo tem o objetivo de apresentar um estudo de caso sobre o consumo das lâmpadas atualmente utilizadas nas salas de aula do Centro Universitário de Belo Horizonte - UniBH, de forma a propor um novo projeto de iluminação, visando a eficiência energética em termos de: redução do consumo de energia e custo de manutenção, maior vida útil das lâmpadas e eficiência luminosa, bem como, melhor conforto visual.
\end{abstract}

Palavras Chaves - Eficiencia Energética. Conforto Visual. Consumo. Custo.

Abstract: The importance of renewable energy and energy efficiency on the global stage has been increased, especially compared to consumption and environment. Some important aspects such as color temperature of lamps, luminaries efficiency, efficiency of the reactor used and luminous efficiency of lamps is extremely important for objectives of a project that aims to achieve results toward energy efficiency. This article aims to present a detailed study of lamps' consumption used today in the University Center Uni-BH in order to propose a better solution to this Center University's energy effiency of consumption and maintenance, lamps' life cycle, efficiency and visual comfort.
Keywords - Energy Efficiency, Comfort Visual, Consumption, Cost.

\section{INTRODUÇÃO}

A importância das energias renováveis no cenário mundial é cada vez maior, principalmente no que diz respeito à energia solar e eólica por serem consideradas limpas do ponto de vista ambiental. Essas fontes entre outras estão gradativamente substituindo os recursos não-renováveis da natureza, como o carvão e o petróleo.

Do ponto de vista da iluminação, comparando a época em que a luz artificial começou a ser utilizada com a dos dias atuais, constata-se que houve um grande avanço na indústria de iluminação do século $\mathrm{XX}$, desde a lâmpada criada por Thomas Edison até os produtos disponíveis atualmente.

Ao contrário do que normalmente se divulga, a lâmpada inventada por Edison não foi a primeira a utilizar a eletricidade, pois no final do século XIX já havia um sistema para iluminação pública composto por dois eletrodos de carvão muito próximos por onde passava uma descarga elétrica. Essa lâmpada era conhecida como lâmpada de arco porque produzia uma luz

\begin{tabular}{l|l|l|l|} 
ISSN: 1984-3151 & www.unibh.br/revistas/exacta/ & Vol. 3 N.․ 2 (2010)
\end{tabular}


intensa muito branca e era utilizada também em faróis de navegação e outras aplicações específicas. O maior problema dessa lâmpada estava justamente na grande quantidade de luz produzida, o que impedia sua utilização em ambientes comerciais ou residenciais. A primeira lâmpada disponível para uso residencial foi a de Edison, por isto considerada como a primeira lâmpada comercial. Ela era constituída de um fio de linha carbonizado em um cadinho hermeticamente fechado, refletindo uma luz amarelada e fraca como a de uma vela e apresentando um rendimento de 1,41 $\mathrm{lm}$ (LUZ, 2010).

Com o avanço da tecnologia pode-se obter diversas formas eficientes e eficazes de se elaborar um projeto luminotécnico, visando a eficiência energética dos sistemas de iluminação. Um bom sistema de iluminação, com o uso adequado de cores no ambiente de trabalho, pode contribuir para criação de uma harmonia no ambiente, reduzir o consumo, evitar a fadiga visual, além de outros problemas relacionados. Uma iluminação correta torna o ambiente mais agradável, permitindo que as pessoas possam desenvolver suas atividades confortavelmente. Desta forma, uma boa iluminação faz com que se eleve o rendimento do trabalho diminuindo-se os erros, contribuindo para mais conforto, bemestar e segurança (ANJOS; MARIBONDO, 2008).

Neste sentido, o presente artigo tem por objetivo avaliar a eficiência do sistema de iluminação de um ambiente real, baseado em um estudo de caso desenvolvido no Centro Universitário de Belo Horizonte - UniBH. Tendo em vista a importância que este fator tem no processo de ensino e aprendizagem, serão apresentados os resultados do estudo luminotécnico de uma sala de aula padrão e as recomendações de melhorias.

$\mathrm{O}$ artigo se encontra dividido em cinco seções, sendo, a primeira, a Introdução; a segunda parte apresenta o problema a ser resolvido; a seguinte aborda a proposta de melhoria com seus benefícios; na quarta parte são discutidos os resultados; e a última apresenta as conclusões.

\section{ESTUDO DE CASO}

Elaborar um estudo voltado para a ergonomia e conforto visual para reduzir o consumo de energia de um estabelecimento não é uma tarefa simples. Este artigo busca apresentar um estudo luminotécnico das salas de aula do Centro Universitário de Belo Horizonte - UniBH, detalhando o consumo de energia atual e a situação a qual se encontra o nível de iluminação do ambiente de modo a propor melhorias para a organização.

\section{II.1 CONCEITOS}

A luz é composta por três cores primárias. A combinação das cores vermelha, verde e azul permite-se obter o branco. A combinação de duas cores primárias produz as cores secundárias: magenta, amarelo e cyan. A partir das três cores primárias dosadas em diferentes quantidades permite-se obter outras cores de luz. Neste contexto a luz é uma radiação eletromagnética capaz de produzir sensação visual. Por outras palavras, é a parte do espectro visível. Trata-se de uma radiação com comprimento de onda entre 380 e $780 \mathrm{~nm}$ (nanometros), sendo uma parte do conhecido espectro de radiação electromagnética.

Quando se fala de luz quente ou luz fria não se refere à maior ou menor quantidade de calor por ela dissipada sob efeito joule, mas sim ao tom de cor que ela dá ao ambiente. A luz quente se refere ao tom mais avermelhado $\mathrm{e}$ produz maior relaxamento. Por outro lado, a luz fria se refere ao tom mais claro proporcionando maior atividade.

A luz proveniente de lâmpadas fluorescentes pode distorcer as cores dos objetos, alimentos e pele das pessoas. Mas isso não é regra geral e independe da sua temperatura de cor. O que define o quanto a lâmpada vai distorcer as cores do que está sob ela é o parâmetro IRC - Índice de Reprodução de Cor. Um bom índice de reprodução de cor para lampâdas fluorescentes é 80. A luz do sol, que é

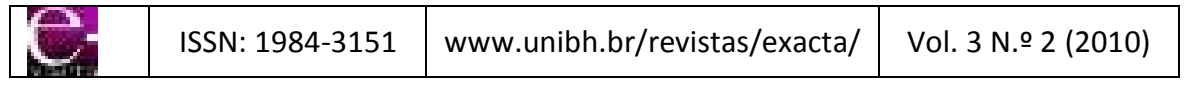


tida como referência perfeita, tem o IRC igual a 100 (CARVALHO, 2010).

Ao se pensar em cálculo luminotécnico, é necessário ter presente quatro critérios principais, que são: a quantidade de luz, o equilíbrio da iluminação, o ofuscamento e, por último, a reprodução de cor (LUZ, 2010). Deve ser dada a maior atenção a cada um desses critérios, pois eles estão diretamente relacionados ao conforto e às necessidades visuais e, portanto, ao bem estar humano.

Ao se iniciar um projeto luminotécnico para um ambiente devem-se realizar opções preliminares, tais como, o tipo de iluminação mais adequada (incandescente, fluorescente, etc.) o tipo de luminária (direta, semi-direta, etc.), sendo que estas opções envolvem aspectos de decoração, tipo do local (sala, escritório, loja, etc.) e as atividades que nele serão desenvolvidas: trabalho bruto de maquinaria, montagem, leitura, etc. (ANJOS; MARIBONDO, 2008).

\section{II.2 Metodologia}

Foi realizada uma análise técnica e econômica da situação atual da Sala de Aula 304 do Bloco A4, Campus Estoril do Centro Universitário de Belo Horizonte - UNI-BH, situado em Belo Horizonte, MG.

Os parâmetros técnicos construtivos da sala em estudo são apresentados na Tabela 1.

Tabela 1 - Parâmetros da Sala 304 do Bloco A4

\begin{tabular}{|l|c|}
\hline \multicolumn{2}{|c|}{ Sala 304 do Bloco A4 } \\
\hline Larg. do Ambiente & $6,5 \mathrm{~m}$ \\
\hline Comp. do Ambiente & $6,9 \mathrm{~m}$ \\
\hline Alt. do Ambiente & $3,8 \mathrm{~m}$ \\
\hline Alt. de Instalação das luminárias & $2,6 \mathrm{~m}$ \\
\hline Plano de Trabalho & $0,75 \mathrm{~m}$ \\
\hline \multicolumn{2}{|c|}{ Índice de Reflexão } \\
\hline Teto & $70 \%$ \\
\hline Parede & $60 \%$ \\
\hline Chão & $50 \%$ \\
\hline
\end{tabular}

Os parâmetros do sistema de iluminação atual são aparesentados na Tabela 2.
Tabela 2 - Especificação técnica do sistema atual.

\begin{tabular}{|l|c|}
\hline \multicolumn{2}{|c|}{ Especificação Técnica } \\
\hline Lâmpada & TLTRS Super $80 / 40 \mathrm{~W} /$ Phillips \\
\hline Diâmetro & $33,5 \mathrm{~mm}$ \\
\hline F. Luminoso & 3250 lumens \\
\hline Vida Mediana & 8000 horas \\
\hline Temp. de cor & $5000^{\circ} \mathrm{K}$ \\
\hline LM/W & 65 \\
\hline Corrente & $0,42 \mathrm{~A}$ \\
\hline Luminária & Simples \\
\hline
\end{tabular}

Pode-se observar que a situação atual está fora dos padrões técnicos. As cores das paredes e do teto não ajudam na capacidade de reflexão de luz, a quantidade de lâmpadas é desnecessária para o ambiente (15 lâmpadas) e não contém luminárias, apenas fixadores de lâmpadas, conforme visto na ilustração representativa exibida na Figura 1.

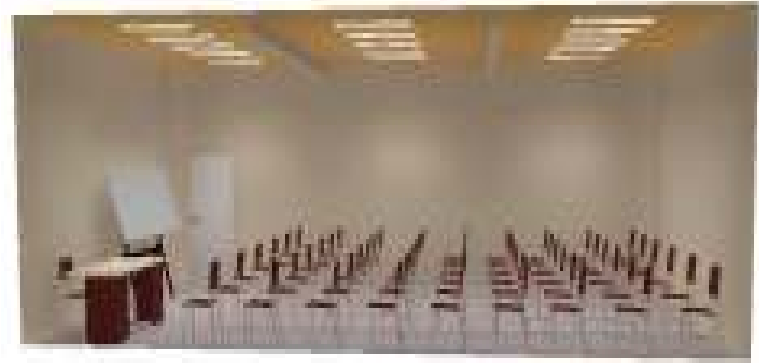

Figura 1 - Representação ilustrativa do layout atual

\section{Melhorias X Benefícios do Projeto}

Para analisar a situação-problema foram usados o método dos Lúmens, ou método o Fluxo Luminoso e os recomendados pela norma técnica NBR5413 (NBR-5113, 1992). O método dos Lúmens é o mais utilizado para os sistemas de iluminação em edificações. O método consiste nos seguintes passos:

- Determinar a iluminância (E):

De acordo com o item 5.3.13 (ESCOLAS) da NBR 5413 da ABNT:

- Salas de aula - 200-300-500

- Foi adotado Iluminancia de 300 lux.

- Determinar a luminária a ser usada: 
Foi adotada a luminária TBS 930 com abas anti-reflexivas (uma lâmpada por luminária).

- Determinar o Fator Local:

Onde:

$$
\mathrm{K}=(\mathrm{CxL}) /[(\mathrm{C}+\mathrm{L}) \times \mathrm{A}]=1,8
$$

C: Comprimento do local $=6,9 \mathrm{~m}$;

L: Largura do local $=6,5 \mathrm{~m}$;

A: Altura do plano de trabalho $=2,6 \mathrm{~m}$.

- Determinar o Coeficiente de Utilização:

Após o cálculo do fator local foi obtido junto com os valores de refletância na tabela do fabricante da luminária um valor de 0,62.

- Determinar o Coeficiente de Manutenção:

Representa a freqüência de limpeza nas peças de vidro e principalmente o entorno da edificação quanto à sujeira do ambiente.

- Ambiente urbano residencial $=0,9$ (NBR5413)

- Determinar o Fluxo Luminoso:

O fluxo luminoso total $[\varphi \mathrm{t}]$, expresso em lumens, que se faz necessário no ambiente, é dado por:

$\Phi \mathrm{t}=(\mathrm{E} \times \mathrm{S}) /(\mathrm{Ku} \times \mathrm{Km})=24.026$ lumens

Onde:

E: Iluminamento desejado (lux) $=300$ lux;

S: Área do local $(\mathrm{m} 2)=44,85 \mathrm{~m}^{2}$;

$\mathrm{Ku}$ : Coeficiente de utilização $=0,62$;

$\mathrm{Km}$ : Coeficiente de manutenção $=0,9$;

Фt: Fluxo luminoso total (lumens) $=24.026$ lumens.

- Determinar o número de lâmpadas:

Divide-se o fluxo luminoso total pelo fluxo luminoso emitido pela lâmpada adotada, é dado por:

$\mathrm{N}=\Phi \mathrm{t} / \Phi \mathrm{i}=$ 9,24 (adotado 9 lâmpadas)

Onde:

$\Phi$ t: Fluxo luminoso total $=24.026$ lumens;

Фi: Fluxo luminoso emitido pela lâmpada $=$ 2600 lumens.
A Tabela 3 especifica o novo sistema de iluminação.

Tabela 3 - Especificação técnica do novo sistema.

\begin{tabular}{|l|c|}
\hline \multicolumn{2}{|c|}{ Especificação Técnica } \\
\hline Lâmpada & $\begin{array}{c}\text { Master TL5 Super 80/ } \\
\text { 28W/Phillips }\end{array}$ \\
\hline Diâmetro & $16 \mathrm{~mm}$ \\
\hline F. Luminoso & 2600 lumens \\
\hline Vida Mediana & 24000 horas \\
\hline Temp. de cor & $3000 / 4000 / 5000^{\circ} \mathrm{K}$ \\
\hline LM/W & 103 \\
\hline Corrente & $0,17 \mathrm{~A}$ \\
\hline Luminária & TBS 930 com abas anti-reflexivas \\
\hline
\end{tabular}

Vale ressaltar que foi proposta a utilização das lâmpadas Máster TL5 do fabricante Philips, que são ideais para iluminação geral de áreas onde exista a necessidade de ótima qualidade de luz aliada a uma excelente economia de energia como, por exemplo, a sala de aula. Estas lâmpadas exigem reatores eletrônicos específicos (Ecotronic), que possuem partida instantânea, reduz até $60 \%$ do consumo de energia comparando com os reatores convencionais, contém baixo fator de potência e são mais leves, compactos e silenciosos (PHILIPS, 2009).

A Figura 2 apresenta o novo layout da sala com o número reduzido de lâmpadas.

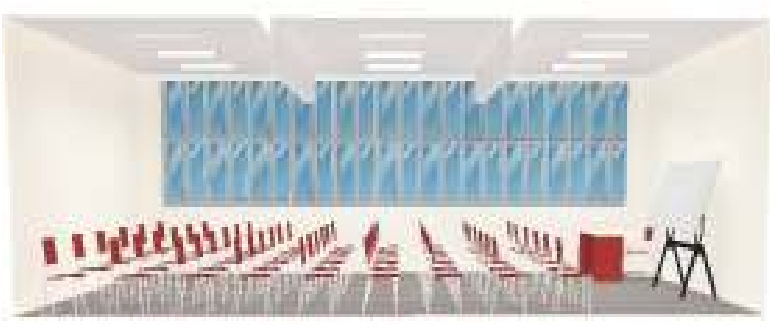

Figura 2 - Demonstração do novo layout com 9 lâmpadas.

\section{ANÁLISE ECONÔMICA}

Nessa seção serão apresentados os resultados econômicos obtidos. Para facilitar o entendimento das análises, a descrição será feita em três partes: a primeira abordará a análise do 
consumo atual confrontando com o novo consumo obtido; a segunda parte abordará o custo de implantação do novo consumo e, a terceira parte, o retorno do investimento do sistema proposto.

\section{IV.1 Consumo Atual X Novo Consumo}

Inicialmente apresentam-se os dados de consumo da sala em estudo. Essas informações servem para facilitar o entendimento dos cálculos realizados. Para o cálculo do consumo atual da sala, foi considerado que a mesma irá funcionar, em média, 10 horas por dia, com todas as lâmpadas acesas. A Tabela 4 apresenta os dados do consumo atual.

Tabela 4 - Demonstração do consumo atual.

\begin{tabular}{|l|c|c|}
\hline \multicolumn{3}{|c|}{ Consumo Atual } \\
\hline Potência Instalada & 15 lâmpadas de $40 \mathrm{~W}$ & $600 \mathrm{~W}$ \\
\hline Kilowatt & $600 \mathrm{~W} / 1000$ & $0,6 \mathrm{~kW}$ \\
\hline Consumo Dário & 10 horas/dia x 0,6 kW & $6 \mathrm{kWh}$ \\
\hline Consumo Mensal & $6 \mathrm{kWh} \times 22$ dias & $\begin{array}{c}132 \\
\mathrm{kWh}\end{array}$ \\
\hline Custo Mensal & $132 \mathrm{kWh} \times \mathrm{R} \$ 0,44^{*}$ & $\mathrm{R} \$$ \\
& $\mathrm{kWh}$ & 58,80 \\
\hline
\end{tabular}

*Informações obtidas junto à concessionária (CEMIG)

A Tabela 5 apresenta os dados do novo consumo do projeto proposto. Deve-se frisar que este consumo foi calculado conforme rege a norma NBR 5413 e também com os dados técnicos do fabricante da Phillips.

Tabela 5 - Demonstração do novo consumo.

\begin{tabular}{|l|c|c|}
\hline \multicolumn{3}{|c|}{ Novo Consumo } \\
\hline $\begin{array}{l}\text { Potência } \\
\text { Instalada }\end{array}$ & 9 lâmpadas de $28 \mathrm{~W}$ & $252 \mathrm{~W}$ \\
\hline Kilowatt & $252 \mathrm{~W} / 1000$ & $0,252 \mathrm{~kW}$ \\
\hline Consumo Dário & 10 horas/dia x 0,252 kW & $2,52 \mathrm{kWh}$ \\
\hline $\begin{array}{l}\text { Consumo } \\
\text { Mensal }\end{array}$ & $2,52 \mathrm{kWh} \times 22$ dias & 55,44 \\
& $\mathrm{kWh}$ \\
\hline Custo Mensal & $55,44 \mathrm{kWh} \times \mathrm{R} \$ 0,44^{*}$ & $\mathrm{R} \$ 24,39$ \\
\hline
\end{tabular}

*Informações obtidas junto à concessionária (CEMIG)

Observa-se, a partir do cálculo do custo mensal, que o novo projeto propiciará economia de aproximadamente $58 \%$.

\section{IV.2 CUSTO DE IMPLANTAÇÃO}

A princípio, o investimento inicial deste novo sistema é elevado, mas como demonstrado na Tabela 6 , o retorno é bastante satisfatório.

Tabela 6 - Demonstração do investimento inicial.

\begin{tabular}{|c|c|c|c|}
\hline \multicolumn{4}{|c|}{ Investimento Inicial } \\
\hline $\begin{array}{c}\text { Compo- } \\
\text { nentes }\end{array}$ & $\begin{array}{c}\text { Valor } \\
\text { Unitário }\end{array}$ & $\begin{array}{c}\mathrm{N}^{\mathrm{o}} . \mathrm{de} \\
\text { Compo- } \\
\text { nentes }\end{array}$ & Valor \\
\hline $\begin{array}{l}\text { Lâmpada } \\
\text { Master } \\
\text { TL 28W }\end{array}$ & $\mathrm{R} \$ 21,27$ & $\begin{array}{c}9 \\
\text { Lâmpadas }\end{array}$ & $\begin{array}{c}\mathrm{R} \$ \\
191,43\end{array}$ \\
\hline $\begin{array}{c}\text { Reator } \mathrm{p} / \mathrm{1} \\
\text { lâmpada } \\
28 \mathrm{~W}\end{array}$ & $\mathrm{R} \$ 58,56$ & 9 Reatores & $\begin{array}{c}\mathrm{R} \$ \\
527,04\end{array}$ \\
\hline $\begin{array}{c}\text { Luminária } \\
\text { TBS } 930\end{array}$ & $\mathrm{R} \$ 110,00$ & $\begin{array}{c}9 \\
\text { Luminárias }\end{array}$ & $\begin{array}{c}\mathrm{R} \$ \\
990,00\end{array}$ \\
\hline \multicolumn{3}{|c|}{ Valor Total: } & $\begin{array}{c}\mathrm{R} \$ \\
1708,47 .\end{array}$ \\
\hline
\end{tabular}

\section{IV.3 RETORNO DO INVESTIMENTO}

Utilizando os cálculos anteriores e confrontando os valores antigos com os valores do novo sistema obtém-se os ganhos que sua implantação pode trazer à Instituição, conforme mostra a Tabela 7.

Tabela 7 - Demonstração do retorno do investimento.

\begin{tabular}{|l|c|c|}
\hline \multicolumn{3}{|c|}{ Retorno do Investimento } \\
\hline $\begin{array}{l}\text { Custo } \\
\text { Investimento }\end{array}$ & Economia mensal (R\$) & $\begin{array}{c}\text { Retorno } \\
\text { (mensal) }\end{array}$ \\
\hline $\mathrm{R} \$ 1708,47$ & $\mathrm{R} \$ 33,90$ & 50 meses. \\
\hline
\end{tabular}

Considerando que as lâmpadas TL5 têm em média vida útil de 24.000 horas (conforme fabricante) e que as lâmpadas fluorescentes universais usadas atualmente têm em média 8.000 horas, obtiveram-se os seguintes valores:

- $\quad$ Trabalho Mensal = 220 horas;

- Trabalho Anual = 2640 horas;

- Lâmpadas Fluorescentes Universais = 8.000 horas / 2640 horas $=3$ Anos de vida útil;

\begin{tabular}{|l|l|l|l|}
\hline C. & ISSN: 1984-3151 & www.unibh.br/revistas/exacta/ & Vol. 3 N. 2 (2010) \\
\hline
\end{tabular}


- Lâmpadas Master TL5 $=24.000$ horas / 2640 horas $=9$ Anos de vida útil;

- Retorno Investimento $=50$ meses $/ 12$ meses = 4,1 Anos;

- Lucratividade $=9$ anos (Lâmpadas TL5) - 4,1 Anos (Investimento) = 5,9 Anos de Economia.

Baseado nos resultados obtidos pelo estudo percebe-se que a sala 304 apresenta uma superestimação na quantidade de lampâdas, o que gera um consumo de energia acima do necessário. A quantidade de luminárias recomendadas como necessárias para o ambiente faria com que houvesse um melhor aproveitamento dos recursos energéticos.

\section{CONCLUSÕES}

Ao realizar este trabalho procurou-se apresentar de forma objetiva uma introdução ao tema proposto: luminotécnica. Nesse sentido, obtevese uma rica gama de pesquisas, contendo assuntos científicos especializados, além de apoio técnico de representantes de equipamentos. Foi possível, a partir das análises feitas, constatar que a Sala de Aula 304 do bloco A4, Campus Estoril, do Centro Universitário de Belo Horizonte - UniBH, apresenta algumas desconformidades ou situações que carecem de correção do ponto de vista de eficiência luminosa.

A sala estudada, durante a noite, apresenta média de iluminância inferior ou no mínimo igual ao estabelecido como parâmetro pela NBR 5413. Tal constatação demonstra que o ambiente tem alta propensão à geração da fadiga visual aos alunos. No ambiente de sala de aula, a iluminação é fator decisivo, facilitando o processo de ensino-aprendizado e reduzindo os níveis de fadiga visual, o que acarreta um melhor rendimento de professores e alunos.

Os dados apresentados são teóricos, obtidos junto aos fabricantes. Para a demonstração da viabilidade e dos principais ganhos obtidos a partir desse estudo teórico, propõem-se a sua implantação em um trabalho subseqüente.

\section{REFERÊNCIAS}

ANJOS, Gilney Christierny Barros do; MARIBONDO, Juscelino de Farias. Estudo luminotécnico em instituições de ensino: análise dos ambientes de sala de aula de um curso de administração, 2008. Disponível em: http://www.ufpi.br/reges/uploads/edicao2/Artigo 8.pdf. Acesso em: 12. Nov. 2010.

CARVALHO, Claudia. LÂMPADAS FLUORESCENTES，2010. Disponível em: http://www.lumearquitetura.com.br/pdf/ed01/ed_ 01_Aula.pdf. Acesso em: 16. nov. 2010.

PHILIPS. Lâmpadas e reatores. Manual da Philips, $2009 . \quad$ Disponível em:. http://www.luz.philips.com. Acesso em: 12. nov. 2010.

LUZ, Jeanine Marchiori da. LUMINOTÉCNICA，2010. Disponível em: http://www.iar.unicamp.br/lab/luz/ld/Livros/Lum inotecnica.pdf. Acesso em: 12. nov. 2010.

NBR-5413 - ILUMINÂNCIA DE INTERIORES, Norma Brasileira Regulamentadora, NBR-5413, 1992. 